\title{
Coronavirus Disease 2019, Prothrombotic Factors, and Venous Thromboembolism
}

\author{
Sam Schulman, MD, $\mathrm{PhD}^{1,2}$ \\ ${ }^{1}$ Department of Medicine, Thrombosis and Atherosclerosis Research \\ Institute, McMaster University, Hamilton, Ontario, Canada \\ 2 Department of Obstetrics and Gynecology, I.M. Sechenov First \\ Moscow State Medical University, Moscow, Russia \\ Semin Thromb Hemost 2020;46:772-776.
}

Coronavirus disease 2019 (COVID-19) is causing devastating morbidity and mortality worldwide. Several studies have shown that the severely ill patients have high or very high Ddimer values, and a hypercoagulable state has been described with, in some cases, development of disseminated intravascular coagulation (DIC). A few reports have indicated that there seems to be a higher incidence of venous thromboembolism than expected in otherwise severely ill patients. In this article, we will discuss the prothrombotic changes observed and to what extent they are specific for COVID-19. The incidence of thromboembolic events will be compared with those reported in sepsis and severe influenza A H1N1. The emphasis is on venous events, which have been the most frequently reported events. Finally, the intensity of pharmacological prophylaxis against venous thromboembolism will be discussed.

The pandemic of COVID-19 is affecting almost every country in the world, with the number of cases tested and found infected exceeding two million, with an overall mortality of approximately $7 \%$ at the time of writing. ${ }^{1}$ There are preliminary reports from China on venous thromboembolism based on a relatively small number of patients. ${ }^{2,3}$ Subsequently, European physicians have reported a higher incidence of thromboembolic events, mainly venous, in patients with COVID-19 pneumonia in the intensive care unit (ICU). ${ }^{4}$ Another manifestation of hypercoagulability is DIC, which was not reported in other than the occasional case in the largest published cohorts. ${ }^{5-7}$ On the other hand, abnormalities in the coagulation tests, meeting previously defined criteria for DIC, were observed during the terminal days in $71 \%$ of nonsurvivors in another cohort from Wuhan, China. ${ }^{8}$

These preliminary findings generate several questions, some which are as follows:

- What are the indicators of hypercoagulability in COVID19 infection?

- Is the hypercoagulability specific for COVID-19 infection?
Address for correspondence Sam Schulman, MD, PhD, Thrombosis Service, HHS-General Hospital, 237 Barton Street East, Hamilton, Ontario L8L 2X2, Canada (e-mail: schulms@mcmaster.ca).

- Does the hypercoagulability result in a higher incidence of thromboembolism than in other patients with severe infection in the ICU?

- If so, is it justified to use higher than standard doses of pharmacological prophylaxis against thromboembolism in these patients?

It should be recognized that the data on this infection are rapidly emerging and that information obtained from countries in Europe or North America might differ from those in China due to variations in ethnic susceptibility, environmental conditions (e.g., pollution and health care resources availability), diagnostic routines, and prophylaxis regimens. Therefore, any assumptions or conclusions drawn in this article can be proven wrong after a short time.

\section{Indicators of Hypercoagulability}

Several reports have documented increased D-dimer levels, ${ }^{5-8}$ and these levels were higher on admission in patients who subsequently had to be treated in the ICU than in patients not requiring intensive care, ${ }^{7}$ as well as higher levels in patients who died in the hospital versus survivors. ${ }^{6} \mathrm{~A}$ meta-analysis of 30 studies and 53,000 patients reported that increased D-dimer was associated with severe COVID19 infection, with a $p$-value of $<0.001 .^{9}$ Tang et al found that the D-dimer level on admission was fourfold higher in patients who did not survive the hospitalization compared with survivors ( $2.12 \mathrm{vs.} 0.61 \mu \mathrm{g} / \mathrm{mL} ; p<0.001) .^{8}$ On days 10 and 14 of hospitalization, the D-dimer values among the nonsurvivors had increased to $\geq 21 \mu \mathrm{g} / \mathrm{mL}$ (which was the upper limit of their detection system). Fibrin degradation products (FDPs) showed a similar pattern.

Guan et al reported that the platelet count was higher in nonsevere than in severe infection $\left(172\right.$ vs. $\left.137.5 \times 10^{9} / \mathrm{L}\right),{ }^{5}$ whereas Huang et al found the opposite (149 vs. $196 \times 10^{9} / \mathrm{L}$ published online May 11, 2020
Issue Theme Maintaining Hemostasis and Preventing Thrombosis in COVID-19 -Part I; Guest Editors: Emmanuel J. Favaloro, PhD, FFSc (RCPA), and Giuseppi Lippi, MD.
Copyright $\odot 2020$ by Thieme Medical Publishers, Inc., 333 Seventh Avenue, New York, NY 10001, USA. Tel: +1(212) 760-0888.
DOI https://doi.org/ 10.1055/s-0040-1710337. ISSN 0094-6176. 
for those without or with ICU care, respectively). ${ }^{7}$ Tang et al observed that in the later stages of their disease, 7 out of 21 nonsurvivors had platelet counts of 50 to $100 \times 10^{9} / \mathrm{L}$ and 5 had $<50 \times 10^{9} / \mathrm{L}$, indicating that there was terminal consumption coagulopathy. ${ }^{8}$ Likewise, the prothrombin time (PT) was in this late stage prolonged by 3 to 6 seconds in 5 and by $>6$ seconds in 10 of the 21 nonsurvivors. ${ }^{6}$ On admission, the difference in median PT between survivors and nonsurvivors was, however, only 2 seconds, ${ }^{8}$ or only 1.5 seconds between those requiring or not requiring ICU care. ${ }^{7}$ The activated partial thromboplastin time was, in the latter study, similar in the two subsets (26.2 and 27.7 seconds for requiring vs. not requiring ICU, respectively), ${ }^{7}$ whereas Tang et al saw a nonsignificant trend in the opposite direction between nonsurvivors and survivors ( 44.8 vs. 41.2 seconds, respectively). ${ }^{8}$

Fibrinogen levels did not differ on admission between survivors and nonsurvivors (4.51 and $5.16 \mathrm{~g} / \mathrm{L}$, respectively), but in the terminal stage, the nonsurvivors demonstrated fibrinogen levels of around $1 \mathrm{~g} / \mathrm{L}^{8}$ Although antithrombin values were generally in the normal range, they were significantly lower among nonsurvivors than survivors on days 7 , 10 , and 14 although not initially. ${ }^{8}$

Taken together, the main initial coagulation abnormality is the elevated D-dimer, which is well recognized as an unspecific marker of hypercoagulability. It seems, however, to give already at an early stage an idea of the severity of the infection and is a prognostic indicator of need for ICU care and of fatal outcome.

Furthermore, there is undoubtedly a pronounced inflammatory response in the severely ill patients with high C-reactive protein (CRP) and cytokines. ${ }^{5,7}$ There is also evidence of endorgan damage to the heart, with high-sensitivity cardiac troponin above 99 th percentile in $31 \%$ of patients requiring ICU care versus $4 \%$ of those not in ICU, and to the liver, with majority of the severe patients demonstrating elevated bilirubin and transaminases, acute kidney injury, and acute respiratory distress syndrome., ${ }^{5,7}$ This constellation raises concern about cytokine storm and, with lymphopenia and thrombocytopenia in the picture, of a possible secondary hemophagocytic lymphohistiocytosis as well. ${ }^{10}$ The inflammatory response will also activate the coagulation system, resulting in thromboinflammation or immunothrombosis. ${ }^{11,12}$ Finally, hypoxia mediated through increased expression of hypoxia-inducible transcription factors can not only increase the inflammatory response but also directly activate platelets and plasma coagulation. ${ }^{13}$ Direct targets include tissue factor (increased expression), protein $\mathrm{S}$ (inhibition), ${ }^{14}$ and plasminogen activator inhibitor type 1 (increased levels). The hypoxia in COVID-19 pneumonia is evident and increases with the severity of the disease.

\section{Specificity of Coagulation Abnormalities}

The inflammatory response is seen in sepsis in general and is associated with DIC and microthrombosis. ${ }^{15}$ The mechanisms include activation of monocytes, neutrophils, platelets, and endothelial cells; expression of tissue factor; release of ultralarge von Willebrand factor multimers; release of cytokines; and generation of neutrophil extracellular traps.
A case report of a patient with severe COVID-19 pneumonia managed with lung transplantation demonstrated hemorrhagic infarctions as well as microthrombosis formation in the lungs. 16

In the former COVID severe acute respiratory syndrome, also known as SARS, there was pronounced fibrin deposition in the lungs, and the virus was shown to induce transcription of the hfgl2 prothrombinase gene, which would generate a procoagulant state. ${ }^{17}$ In animal models, there was evidence of increased expression in the urokinase pathway involving pro- and antifibrinolytic genes, and in proteomics analyses of the lung, there was increased expression of fibrin and factor VIII. ${ }^{18}$ Lethal doses (but not sublethal) led to increased levels of plasminogen peptides associated with increased urokinase activity. These changes can explain some of the lung pathology but do not necessarily induce venous thromboembolism.

There is no clear evidence for a different mechanism for the activation of coagulation in COVID-19 pneumonia compared with severe sepsis, except for the fact that hypoxia may be worse in the former.

\section{Incidence of Thromboembolism Compared with Other Patients with Severe Infection in the ICU}

Whether SARS-CoV-1, the microorganism causing SARS, triggered increased PE is difficult to know since the focus of the medical staff was on treatment of respiratory failure. Computed tomography (CT) of the chest is not performed to diagnose pulmonary embolism (PE), and CT of the pulmonary arteries was rarely performed and was, at the time, performed mainly using less sophisticated equipment than today and therefore may have missed subsegmental emboli.

In the XPRESS (Xigris and Prophylactic Heparin Evaluation in Severe Sepsis) study, 1,994 patients with sepsis received activated protein $C$ and were randomized to prophylaxis against venous thromboembolism with heparin or placebo. There were events of venous thromboembolism during the study period with a similar incidence in patients who received unfractionated heparin (5.6\%), low-molecular-weight heparin (LMWH) $(5.9 \%)$, or placebo (7.0\%). ${ }^{19}$ Most of the events were found on screening for deep vein thrombosis (DVT). The PROTECT (PROphylaxis for ThromboEmbolism in Critical Care Trial) trial randomized 3,764 critically ill patients to dalteparin or unfractionated heparin, and overall the incidence of venous thromboembolism was similar. ${ }^{20}$ Dalteparin (5,000 units once daily) was associated with a lower risk of PE ( 1.3 vs. $2.3 \%$; hazard ratio: 0.51 ; $95 \%$ confidence interval: 0.30 0.88 ), and the risk of DVT was $7.4 \%$, of which $5.1 \%$ were proximal and mainly detected by screening with ultrasound.

An early review of findings on CT in patients with COVID19 did not mention PE, but it should be noted that only CT chest was performed, and without performing CT pulmonary angiogram, emboli could have been missed. ${ }^{21}$

In another study from China, 81 patients treated in the ICU were examined through ultrasonography of the leg veins and CT of the chest. ${ }^{3}$ Of the 81 patients, 20 (25\%) developed DVT. The value of D-dimer was much higher in those who developed 
thrombosis than among those who did not $(5.2 \pm 3.0$ vs. $0.8 \pm 1.2 \mu \mathrm{g} / \mathrm{mL} ; p<0.001)$, but it is not stated when samples were taken. Furthermore, it is not clear how many, if any, of the patients received prophylaxis against thrombosis.

Colleagues in Italy, Spain, and New York City have diagnosed DVT and PE in higher proportions of patients (personal communications), but these are just preliminary, though reliable, observations.

At three hospitals in the Netherlands, all patients with COVID-19 pneumonia admitted to the ICU were evaluated for venous and arterial thromboembolic complications. ${ }^{4}$ All patients received prophylaxis with a standard or increased dose of LMWH. It appears from the report that the investigators only performed objective diagnostic imaging in patients with symptoms, raising suspicion of a thromboembolic event, and among those, the composite outcome of all thromboembolism was as high as $31 \%$ or a total of 31 cases. Of those, 25 had PE, but there is no information regarding the location of the emboli and how many of those were only subsegmental. Of the three cases with DVT, two were catheter-related upper extremity thrombosis and one was a proximal leg vein thrombosis. Stroke was verified in three patients, presumably with evidence of acute infarct. These findings are difficult to interpret regarding PE due to the paucity of information and also the difficulty in finding comparable studies on other infections investigated in the same way. A symptomatic DVT rate of 1 per 184 or of catheter-related thrombosis of 2 per 184 is not remarkable.

In a recent review of 17 studies on atrial fibrillation in patients with sepsis, new-onset atrial fibrillation was diagnosed in 2 to $48 \%$ of patients, and new strokes were identified in 0 to $5 \%$ of those. ${ }^{22}$ Stroke was also, albeit at a slightly lower incidence, diagnosed in sepsis patients remaining in sinus rhythm.

Several retrospective studies showed a high incidence of thromboembolic events in patients hospitalized for the influenza A H1N1, as shown in - Table 1. 23-27 Among patients requiring critical care or with fatal outcome, the incidence was 25 to $70 \%$, which was dominated by PE without DVT. For patients admitted to the hospital but not requiring ICU care, the incidence was 0 to $6 \%$. The mechanisms behind hypercoagulability in this infection have been reviewed by Lippi et al. ${ }^{28}$

The data so far are thus not conclusive regarding a truly increased incidence of clinically important thromboembolic events in patients with COVID-19. There might, however, be a predominance of pulmonary artery thrombi rather than embolism from the leg veins due to the inflammatory reaction in the lungs causing profound local hypercoagulability, as suggested by the H1N1 data ( - Table $\mathbf{1}$ ) and as also reviewed recently. $^{29}$

\section{Dose of Low-Molecular-Weight Heparin: Standard or Increased?}

In most of the studies from China, it is not reported whether, and in such cases how many, patients received chemoprophylaxis against venous thromboembolism and at what dose. Cui et al reported that when patients with DVT were

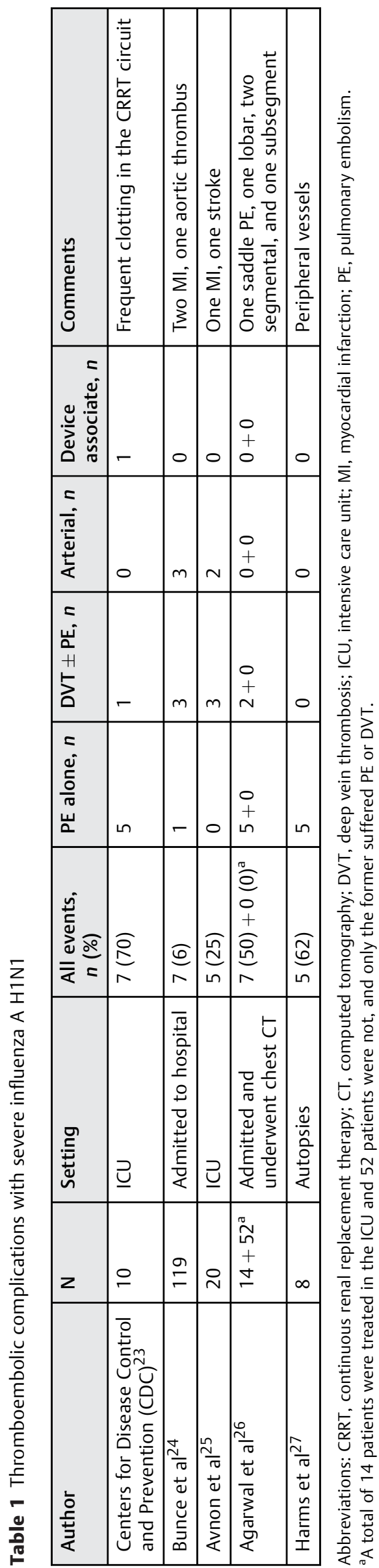


started on therapeutic anticoagulation, the D-dimer values decreased. $^{3}$ In a Dutch study, two of the three hospitals increased the prophylactic dose of nadroparin during the study period, but it is unclear whether this change led to a reduced incidence of thromboembolism. ${ }^{4}$

The American Society of Hematology has on its website a recommendation to provide the usual thromboprophylaxis unless there is a clear indication for treatment doses, ${ }^{30}$ and a similar indication (i.e., enoxaparin 40-60 mg every day) has also been recently endorsed by the Italian National Medicines Agency (AIFA).

There have not been any reports of a pattern of increased bleeding complications in patients with severe COVID-19 infection despite a tendency to develop thrombocytopenia. Hemoptysis was reported in 4 of 173 patients with severe disease and in 6 of 926 with nonsevere disease. ${ }^{5}$ It is therefore reasonable to assume that there is, in general, hyper- rather than hypocoagulability. Nevertheless, this is the case for many other patients admitted to hospital and ICU, and increased doses of heparin or LMWH are not recommended for those. Increased doses of anticoagulants may always carry a risk of more bleeding events. The only way to resolve this is by performing randomized trials. Observational studies will be fraught with a large number of confounders in view of the variety of intensive care procedures performed and the increasing number of experimental therapies introduced to manage severe COVID-19 pneumonia.

During the few days between submission and receiving the page proofs, two additional reports of high incidence of venous thromboembolism despite prophylaxis have emerged. In a prospective study of 150 consecutive patients with COVID-19 admitted to ICU at two French hospitals, CT of pulmonary arteries was done in case of deteriorating condition or spike in D-dimer. ${ }^{31}$ Out of 100 examined, 25 (25\%) had PE of which only 3 were subsegmental, and 3 patients had DVT. All patients were receiving prophylactic $(n=105)$ or therapeutic $(n=45)$ anticoagulation. A second Dutch cohort included 198 admitted patients with 74 (37\%) in ICU. ${ }^{32}$ Standard or intermediate LMWH was routine prophylaxis against DVT. After a median observation time of 5 days, 33 patients (17\%) were diagnosed with PE ( $n=11$, of which 2 subsegmental) or DVT $(n=22)$. Most of the events $(n=29)$ were in ICU patients and 21 of the 33 events were symptomatic with the remainder found through screening for DVT. These data support unusually high incidence of venous thromboembolism despite LMWH prophylaxis.

\section{Conclusion}

In severe COVID-19 pneumonia, there are several changes in the prothrombotic direction, and this can be explained by profound inflammatory response as well as hypoxia. Many of the changes are similar to what is otherwise seen in sepsis, but hypoxia is likely an aggravating factor in COVID-19 pneumonia. This may result in an increased incidence of pulmonary thrombosis, which may similarly have been the case in critically ill patients with influenza A H1N1. Venous thromboembolism despite chemoprophylaxis in patients in ICU is not a new phenomenon, and it is not clear whether this occurs more often with COVID-19. Thus, it seems premature to recommend intermediate or therapeutic doses of heparin or LMWH until we have evidence from randomized clinical trials.

\section{Conflict of Interest}

Dr. Schulman reports grants and personal fees from Octapharma and Boehringer Ingelheim, and grants from Alnylam, Bayer, Bristol-Myers Squibb, Daiichi Sankyo, and grants from Sanofi, outside the submitted work.

\section{References}

1 Center for Systems Science and Engineering (CSSE) at Johns Hopkins University. (JHU). Coronavirus COVID-19 Global Cases. Available at: www.arcgis.com/apps/opsdashboard/index.html\#/ bda7594740fd40299423467b48e9ecf6. Accessed April 13, 2020

2 Xie Y, Wang X, Yang P, Zhang S. COVID-19 complicated by acute pulmonary embolism. Radiol Cardiothorac Imaging 2020;2(02): e200067

3 Cui S, Chen S, Li X, Liu S, Wang F. Prevalence of venous thromboembolism in patients with severe novel coronavirus pneumonia. J Thromb Haemost 2020 (e-pub ahead of print). Doi: 10.1111/ jth.14830

4 Klok FA, Kruip MJHA, van der Meer NJM, et al. Incidence of thrombotic complications in critically ill ICU patients with COVID-19. Thromb Res 2020 (e-pub ahead of print) . Doi: 10.1016/j.thromres.2020.1004.1013

5 Guan WJ, Ni ZY, Hu Y, et al; China Medical Treatment Expert Group for Covid-19. Clinical characteristics of coronavirus disease 2019 in China. N Engl J Med 2020 (e-pub ahead of print) . Doi: 10.1056/NEJMoa2002032

6 Zhou F, Yu T, Du R, et al. Clinical course and risk factors for mortality of adult inpatients with COVID-19 in Wuhan, China: a retrospective cohort study. Lancet 2020;395(10229):1054-1062

7 Huang C, Wang Y, Li X, et al. Clinical features of patients infected with 2019 novel coronavirus in Wuhan, China. Lancet 2020;395 (10223):497-506

8 Tang N, Li D, Wang X, Sun Z. Abnormal coagulation parameters are associated with poor prognosis in patients with novel coronavirus pneumonia. J Thromb Haemost 2020;18(04):844-847

9 Ma C, Gu J, Hou P, et al. Incidence, clinical characteristics and prognostic factor of patients with COVID-19: a systematic review and meta-analysis. MedRxiv 2020 (e-pub ahead of print) . Doi: 10.1101/2020.03.17.20037572

10 Mehta P, McAuley DF, Brown M, Sanchez E, Tattersall RS, Manson JJ; HLH Across Speciality Collaboration, UK. COVID-19: consider cytokine storm syndromes and immunosuppression. Lancet 2020;395(10229):1033-1034

11 Jackson SP, Darbousset R, Schoenwaelder SM. Thromboinflammation: challenges of therapeutically targeting coagulation and other host defense mechanisms. Blood 2019;133(09):906-918

12 Gaertner F, Massberg S. Blood coagulation in immunothrombosisat the frontline of intravascular immunity. Semin Immunol 2016; 28(06):561-569

13 Gupta N, Zhao YY, Evans CE. The stimulation of thrombosis by hypoxia. Thromb Res 2019;181:77-83

14 Prchal JT. Hypoxia and thrombosis. Blood 2018;132(04):348-349

15 Iba T, Levi M, Levy JH. Sepsis-induced coagulopathy and disseminated intravascular coagulation. Semin Thromb Hemost 2020;46 (01):89-95

16 Luo W, Yu H, Gou J, et al. Clinical pathology of critical patient with novel coronavirus pneumonia (COVID-19). Preprints 2020, 2020020407 
17 Han M, Yan W, Huang Y, et al. The nucleocapsid protein of SARS$\mathrm{CoV}$ induces transcription of hfgl2 prothrombinase gene dependent on C/EBP alpha. J Biochem 2008;144(01):51-62

18 Gralinski LE, Bankhead A III, Jeng S, et al. Mechanisms of severe acute respiratory syndrome coronavirus-induced acute lung injury. MBio 2013;4(04):e00271-13

19 Shorr AF, Williams MD. Venous thromboembolism in critically ill patients. Observations from a randomized trial in sepsis. Thromb Haemost 2009;101(01):139-144

20 Cook D, Meade M, Guyatt G, et al; PROTECT Investigators for the Canadian Critical Care Trials Group and the Australian and New Zealand Intensive Care Society Clinical Trials Group. Dalteparin versus unfractionated heparin in critically ill patients. $\mathrm{N}$ Engl J Med 2011;364(14):1305-1314

21 Ye Z, Zhang Y, Wang Y, Huang Z, Song B. Chest CT manifestations of new coronavirus disease 2019 (COVID-19): a pictorial review. Eur Radiol 2020 (e-pub ahead of print) . Doi: 10.1007/s00330-02006801-0

22 Aibar J, Schulman S. New-onset atrial fibrillation in sepsis: a narrative review. Semin Thromb Hemost 2020. In press

23 Centers for Disease Control and Prevention (CDC). Intensive-care patients with severe novel influenza A (H1N1) virus infection Michigan, June 2009. MMWR Morb Mortal Wkly Rep 2009;58 (27):749-752

24 Bunce PE, High SM, Nadjafi M, Stanley K, Liles WC, Christian MD. Pandemic H1N1 influenza infection and vascular thrombosis. Clin Infect Dis 2011;52(02):e14-e17
25 Avnon LS, Munteanu D, Smoliakov A, Jotkowitz A, Barski L. Thromboembolic events in patients with severe pandemic influenza A/H1N1. Eur J Intern Med 2015;26(08):596-598

26 Agarwal PP, Cinti S, Kazerooni EA. Chest radiographic and CT findings in novel swine-origin influenza A (H1N1) virus (S-OIV) infection. AJR Am J Roentgenol 2009;193(06):1488-1493

27 Harms PW, Schmidt LA, Smith LB, et al. Autopsy findings in eight patients with fatal H1N1 influenza. Am J Clin Pathol 2010;134 (01):27-35

28 Lippi G, Franchini M, Favaloro EJ. Influenza and cardiovascular disease: does swine-origin, $2009 \mathrm{H} 1 \mathrm{~N} 1 \mathrm{flu}$ virus represent a risk factor, an acute trigger, or both? Semin Thromb Hemost 2010;36 (01):49-58

29 Marongiu F, Mameli A, Grandone E, Barcellona D. Pulmonary thrombosis: a clinical pathological entity distinct from pulmonary embolism? Semin Thromb Hemost 2019;45(08):778-783

30 American Society of Hematology. COVID-19 and VTE/Anticoagulation: Frequently Asked Questions. Available at: www.hematology.org/covid-19/covid-19-and-vte-anticoagulation. Accessed April 13, 2020

31 Helms J, Tacquard C, Severac F, et al. High risk of thrombosis in patients in severe SARS-CoV-2 infection: a multicenter prospective cohort study. Intensive Care Medicine 2020 (epub ahead of print) doi: 10.1007/s00134-020-06062-x

32 Middeldorp S, Coppens M, van Haaps TF, et al. Incidence of venous thromboembolism in hospitalized patients with COVID-19. JThromb Haemost 2020. doi: 10.1111/jth.14888. [Epub ahead of print] 